\title{
A note on vortices from Lorentz-violating models
}

\author{
Lucas Sourrouille ${ }^{a, b}$ \\ ${ }^{a}$ Departamento de Física, Universidade Federal do Maranhão, \\ 65085-580, São Luís, Maranhão, Brazil \\ ${ }^{b} C M C C$, Universidade Federal do ABC, Santo André, SP, Brazil \\ lsourrouille@yahoo.es
}

April 15, 2014

\begin{abstract}
We consider two self-dual abelian Higgs systems obtained from Lorentz breaking symmetry models by dimensional reduction. For the first model, we show that the selfdual equations are identical to those of Nielsen-Olesen vortices. Also, we show that our vortices have electric charge. In the second case we show that self-dual Chern-SimonsHiggs vortices without electric charge are possible.
\end{abstract}

Keywords:Chern-Simons-like gauge theory, Topological solitons, Lorentz symmetry violation.

pacs: $11.10 . \mathrm{Kk}, 11.10 . \mathrm{Lm}, 11.27 .+\mathrm{d}, 12.60 . \mathrm{i}, 11.30 . \mathrm{Cp}$

\section{Introduction}

The possible violation of Lorentz invariance has recently received a lot of attention as a candidate for the Planck scale physics [1. The large range of existing phenomenological and experimental activities stems from the application of effective field theory [2] and the construction of the Standard-Model Extension (SME) [3, 4] to catalogue and predict observable effects. The gauge sector of the SME include CPT-even and CPT-odd terms. The CPT-odd, usully called the Carroll-Field-Jackiw term[5], consist on four parameters, that engenders a parity-odd and birefringent electrodynamics, coupled to Chern-Simons dynamics. The CPT-even photon sector is composed by fourth-rank tensor, with Riemann tensor symmetries and a double null trace, which is coupled to a gauge term $F^{\kappa \lambda} F^{\mu \nu}$. These models have been studied in different contexts, such as radiative corrections [6], nontrivial spacetime topology [7], causality [8], supersymmetry 
[9], the cosmic microwave background [10], vacuum Cherenkov radiation[11, general relativity [12] and soliton solutions, like monopoles[13] and vortices [14, 15, 16].

On the other hand, it is well known that the abelian Higgs model [17] in $(2+1)$ dimensions, support topological vortex solutions of finite energy. With a specific choice of coupling constants, minimum energy vortex configurations satisfy first order differential equations [18]. This model admits and $N=2$ supersymmetric extension providing a connection between BPS equations and supersymmetry [19]. Also the $(2+1)$-dimensional matter field interacting with gauge fields whose dynamics is governed by a ChernSimons term support soliton solutions [20]. When self-interactions are suitably chosen, vortex configurations satisfy the Bogomolnyi-type equations with a specific sixth order potential [21]. Another important feature of the ChernSimons gauge field is that inherits its dynamics from the matter fields to which it is coupled, so it may be either relativistic [21] or non-relativistic [22]. In addition the soliton solutions are of topological and non-topological nature [23].

The purpose of this letter is the study of the self-dual vortices in two Lorentz-violating models. In the first section we will analyze the self-dual equations of a two dimensional model obtained from the CPT-even Lorentz-violating term, coupled to matter field, by dimensional reduction. We will show that this model support self-dual vortex solutions with electric charge. In particular, we will show that the self-duality equations are identical to those of the Nielsen-Olesen vortices. The second section is dedicated to explore the dimensional reduction of the $C P T$-odd Lorentz-violating term couple to a Higgs field. We will show, here, that it is possible to obtain exactly the same Bogomolnyi equations as the relativistic Chern-Simons-Higgs model. The novelty is that our vortices have no electric charge unlike the Chern-Simons-Higgs vortex solutions.

\section{Nielsen-Olesen vortices with electric charge from $C P T$-even Lorentz-violating model}

Let us start by considering the $(3+1)$-dimensional $C P T$-even Lorentz-violating model governed by the action [3],

$$
S=\int d^{4} x\left(-\frac{1}{4} k_{\kappa \lambda \mu \nu} F^{\kappa \lambda} F^{\mu \nu}-A^{\nu} J_{\nu}\right),
$$

where the metric tensor is $g^{\mu \nu}=(1,-1,-1,-1)$. The coupling $k_{\kappa \lambda \mu \nu}$ is real and dimensionless. It has the symmetries of the Riemann tensor and zero double trace, so it contains 19 independent real components. The field strength $F^{\mu \nu}$ is defined by

$$
F^{\mu \nu}=\partial^{\mu} A^{\nu}-\partial^{\nu} A^{\mu}
$$

and the term $A^{\nu} J_{\nu}$ represents the coupling between the gauge field and an external current.

The equation of motion arising from the Lagrangian (11) is

$$
k_{\mu \alpha \beta \gamma} \partial^{\alpha} F^{\beta \gamma}+J^{\mu}=0
$$


In the following we will consider $(3+1)$ dimensional $C P T$-even Lorentz-violating model coupled to Higgs $(\phi)$ matter

$$
S=\int d^{4} x\left(-\frac{1}{4} k_{\kappa \lambda \mu \nu} F^{\kappa \lambda} F^{\mu \nu}+\left|D_{\mu} \phi\right|^{2}-V(|\phi|)\right)
$$

The covariant derivative is defined as $D_{\mu}=\partial_{\mu}+i e A_{\mu}$ and $V(|\phi|)$ is a self-interacting potential. Since, we will analyze the dimensional reduction of the model (4), it is convenient to expand the first term of the action,

$$
\begin{aligned}
S_{C P T}= & -\frac{1}{4} \int d^{4} x k_{\kappa \lambda \mu \nu} F^{\kappa \lambda} F^{\mu \nu} \\
= & -2 \int d^{4} x\left(k_{0 i 0 i}\left(F^{0 i}\right)^{2}+k_{i j i j}\left(F^{i j}\right)^{2}+k_{0 j 0 i} F^{0 j} F^{0 i}+\right. \\
& \left.k_{1 \mu 1 \nu} F^{1 \mu} F^{1 \nu}+k_{2 \mu 2 \nu} F^{2 \mu} F^{2 \nu}+k_{3 \mu 3 \nu} F^{3 \mu} F^{3 \nu}+k_{0 i j \kappa} F^{0 i} F^{j \kappa}\right),
\end{aligned}
$$

where

$$
\begin{aligned}
k_{0 i 0 i}\left(F^{0 i}\right)^{2} & =k_{0101}\left(F^{01}\right)^{2}+k_{0202}\left(F^{02}\right)^{2}+k_{0303}\left(F^{03}\right)^{2} \\
k_{i j i j}\left(F^{i j}\right)^{2} & =k_{1212}\left(F^{12}\right)^{2}+k_{1313}\left(F^{13}\right)^{2}+k_{2323}\left(F^{23}\right)^{2} \\
k_{0 j 0 i} F^{0 j} F^{0 i} & =k_{0102} F^{01} F^{02}+k_{0103} F^{01} F^{03}+k_{0203} F^{02} F^{03} \\
k_{1 \mu 1 \nu} F^{1 \mu} F^{1 \nu} & =k_{1012} F^{10} F^{12}+k_{1013} F^{10} F^{13}+k_{1213} F^{12} F^{13} \\
k_{2 \mu 2 \nu} F^{2 \mu} F^{2 \nu} & =k_{2023} F^{20} F^{23}+k_{2021} F^{20} F^{21}+k_{2123} F^{21} F^{23} \\
k_{3 \mu 3 \nu} F^{3 \mu} F^{3 \nu} & =k_{3031} F^{30} F^{31}+k_{3032} F^{30} F^{32}+k_{3132} F^{31} F^{32} \\
k_{0 i j \kappa} F^{0 i} F^{j \kappa} & =2 k_{0123} F^{01} F^{23}+2 k_{0312} F^{03} F^{12}+2 k_{0231} F^{02} F^{31}
\end{aligned}
$$

We will study the soliton solution in a $(2+1)$-dimensional model, obtained from (44) via dimensional reduction. In order to carry out this procedure it is natural to consider a dimensional reduction of the action by suppressing dependence on the third spacial coordinate and renaming $A_{3}$ as $N$. Then, the action (41) becomes

$$
S_{(2+1)}=\int d^{3} x\left(S_{r C P T}+\left|D_{0} \phi\right|^{2}-\left|D_{i} \phi\right|^{2}-e^{2} N^{2}|\phi|^{2}-V(|\phi|)\right),
$$

where $S_{r C T P}$ is the reduced $C P T$-even Lorentz-violating action,

$$
\begin{aligned}
S_{r C P T}= & -2 \int d^{3} x\left(k_{0 i 0 i}\left(F^{0 i}\right)^{2}+k_{\mu 3 \mu 3}\left(\partial^{\mu} N\right)^{2}+k_{1212}\left(F^{12}\right)^{2}+k_{\mu \nu \mu 3} F^{\mu \nu} \partial^{\mu} N\right. \\
& +k_{0312} F^{12}\left(\partial^{0} N\right)+k_{0 i j 3} F^{0 i}\left(\partial^{j} N\right)+k_{i 0 i j} F^{i 0} F^{i j} \\
& \left.+k_{0102} F^{01} F^{02}+k_{303 i}\left(\partial^{0} N\right)\left(\partial^{i} N\right)+k_{3132}\left(\partial^{1} N\right)\left(\partial^{2} N\right)\right),
\end{aligned}
$$

with $(i, j=1,2)$ and $(\mu, \nu=0,1,2)$. 
Here, we are interested in time-independent soliton solutions that ensure the finiteness of the action (77). These are the stationary points of the energy which for the static field configuration reads

$$
\begin{aligned}
E_{(2+1)}= & \int d^{2} x\left[2 \left(k_{0 i 0 i}\left(\partial^{i} A^{0}\right)^{2}+k_{i 3 i 3}\left(\partial^{i} N\right)^{2}+k_{1212}\left(F^{12}\right)^{2}+k_{i j i 3} F^{i j} \partial^{i} N\right.\right. \\
& +k_{i 0 i 3} \partial^{i} A^{0} \partial^{i} N+k_{0 i j 3}\left(-\partial^{i} A^{0}\right)\left(\partial^{j} N\right)+k_{i 0 i j}\left(\partial^{i} A^{0}\right) F^{i j} \\
& \left.+k_{0102}\left(\partial^{1} A^{0}\right)\left(\partial^{2} A^{0}\right)+k_{3132}\left(\partial^{1} N\right)\left(\partial^{2} N\right)\right)+e^{2}|\phi|^{2}\left(N^{2}-A_{0}^{2}\right) \\
& \left.+\left|\left(D_{1} \pm i D_{2}\right) \phi\right|^{2} \mp e F_{12}|\phi|^{2} \pm \frac{1}{2} \epsilon^{i j} \partial_{i} J_{j}+\frac{\lambda}{4}\left(|\phi|^{2}-v^{2}\right)^{2}\right],
\end{aligned}
$$

where we have used the fundamental identity

$$
\left|D_{i} \phi\right|^{2}=\left|\left(D_{1} \pm i D_{2}\right) \phi\right|^{2} \mp e F_{12}|\phi|^{2} \pm \epsilon^{i j} \partial_{i} J_{j}
$$

The potential term has been chosen so that it coincides with the symmetry breaking potential of the Higgs model

$$
V(|\phi|)=\frac{\lambda}{4}\left(|\phi|^{2}-v^{2}\right)^{2}
$$

The term $\int d^{2} x \frac{1}{2} \epsilon^{i j} \partial_{i} J_{j}$ is a surface term and may be dropped with the hypothesis that

$$
\begin{array}{r}
\left.\phi\right|_{|x|=\infty}=v e^{i \alpha(\theta)} \\
\left.A_{i}\right|_{|x|=\infty}=\frac{i}{e} \partial_{i}(\log \phi)
\end{array}
$$

This condition also implies that the covariant derivative must vanish asymptotically, which fixes the behavior of the gauge field $A_{i}$. Then we have

$$
\Phi=\int d^{2} x F_{12}=\oint_{|x|=\infty} A_{i} d x^{i}=2 \pi n
$$

where $n$ is a topological invariant which takes only integer values.

The formula (9) may be rewritten as a sum of square terms which are bounded below by a multiple of the magnitude of the magnetic flux. To achieve this we can regroup the terms of (9) as follows,

$$
k_{0 i 0 i}\left(\partial^{i} A^{0}\right)^{2}+k_{i 3 i 3}\left(\partial^{i} N\right)^{2}+k_{i 0 i 3} \partial^{i} A^{0} \partial^{i} N=k_{0 i 0 i}\left(\partial^{i} A^{0} \pm \partial^{i} N\right)^{2},
$$

where, we have chosen $k_{0 i 0 i}=k_{i 3 i 3}= \pm \frac{1}{2} k_{i 0 i 3}$. In addition, if we choose $\pm k_{i 0 i j}=k_{i j i 3}$, we have

$$
k_{i j i 3} F^{i j} \partial^{i} N+k_{i 0 i j} \partial^{i} A^{0} F^{i j}=k_{i 0 i j} F^{i j}\left(\partial^{i} A^{0} \pm \partial^{i} N\right)
$$

Since

$$
k_{0 i j 3}\left(-\partial^{i} A^{0}\right)\left(\partial^{j} N\right)=-k_{0123}\left(\partial^{1} A^{0}\right)\left(\partial^{2} N\right)+k_{0213}\left(\partial^{2} A^{0}\right)\left(\partial^{1} N\right),
$$


we can choose, $-k_{0123}= \pm k_{3132}$ and $k_{0102}= \pm k_{0213}$, so that

$$
\begin{aligned}
& k_{3132}\left(\partial^{1} N\right)\left(\partial^{2} N\right)-k_{0123}\left(\partial^{1} A^{0}\right)\left(\partial^{2} N\right)=k_{0123} \partial^{2} N\left(-\partial^{1} A^{0} \mp \partial^{1} N\right) \\
& k_{0213}\left(\partial^{2} A^{0}\right)\left(\partial^{1} N\right)+k_{0102}\left(\partial^{2} A^{0}\right)\left(\partial^{1} A^{0}\right)=k_{0102} \partial^{2} A^{0}\left(\partial^{1} A^{0} \pm \partial^{1} N\right)
\end{aligned}
$$

Finally, we can write the following identity:

$$
\begin{aligned}
& 2 k_{1212}\left(F^{12}\right)^{2} \mp e F^{12}|\phi|^{2}+\frac{\lambda}{4}\left(|\phi|^{2}-v^{2}\right)^{2}= \\
& \frac{\kappa}{2}\left[F^{12} \mp \frac{e}{\kappa}\left(|\phi|^{2}-v^{2}\right)\right]^{2}+\left(2 k_{1212}-\frac{\kappa}{2}\right)\left(F^{12}\right)^{2}+ \\
& \left(\frac{\lambda}{4}-\frac{e^{2}}{2 \kappa}\right)\left(|\phi|^{2}-v^{2}\right)^{2} \mp e v^{2} F^{12}
\end{aligned}
$$

If the coupling constant $\lambda$ and $\kappa$ are chosen such that

$$
\begin{gathered}
\lambda=\frac{e^{2}}{2 k_{1212}} \\
\kappa=4 k_{1212},
\end{gathered}
$$

the expression (18) takes the simple form

$$
\begin{aligned}
& 2 k_{1212}\left(F^{12}\right)^{2} \mp e F^{12}|\phi|^{2}+\frac{\lambda}{4}\left(|\phi|^{2}-v^{2}\right)^{2}= \\
& 2 k_{1212}\left[F^{12} \mp \frac{e}{4 k_{1212}}\left(|\phi|^{2}-v^{2}\right)\right]^{2} \mp e v^{2} F^{12}
\end{aligned}
$$

Using these identities, the energy functional (9) becomes

$$
\begin{aligned}
E_{(2+1)}= & \int d^{2} x\left[2 \left(k_{0 i 0 i}\left(\partial^{i} A^{0} \pm \partial^{i} N\right)^{2}+k_{i 0 i j} F^{i j}\left(\partial^{i} A^{0} \pm \partial^{i} N\right)\right.\right. \\
& +k_{0123} \partial^{2} N\left(-\partial^{1} A^{0} \mp \partial^{1} N\right)+k_{0102} \partial^{2} A^{0}\left(\partial^{1} A^{0} \pm \partial^{1} N\right) \\
& \left.+k_{1212}\left[F^{12} \mp \frac{e}{4 k_{1212}}\left(|\phi|^{2}-v^{2}\right)\right]^{2}\right)+e^{2}|\phi|^{2}\left(N^{2}-A_{0}^{2}\right) \\
& \left.+\left|\left(D_{1} \pm i D_{2}\right) \phi\right|^{2} \mp e v^{2} F^{12}\right]
\end{aligned}
$$

If we choose $A_{0}=\mp N$, then the fields $A_{0}$ and $N$ are decoupled to the fields $A_{i}$ and $\phi$ and we obtain an expression for the energy similar to the well known energy for the Maxwell-Higgs model

$$
E_{(2+1)}=\int d^{2} x\left(2 k_{1212}\left[F^{12} \mp \frac{e}{4 k_{1212}}\left(|\phi|^{2}-v^{2}\right)\right]^{2}+\left|\left(D_{1} \pm i D_{2}\right) \phi\right|^{2} \mp e v^{2} F^{12}\right),
$$

Thus, the energy (22) is reduced to a sum of square terms which are bounded below by a multiple of the magnitude of the magnetic flux(for positive flux we choose the lower signs, and for negative flux we choose the upper signs):

$$
E_{(2+1)} \geq e v^{2}|\Phi|
$$


The bound is saturated by fields satisfying the first-order Bogomolnyi [18] self-duality equations:

$$
\begin{aligned}
& \left(D_{1} \pm i D_{2}\right) \phi=0 \\
& F^{12} \mp \frac{e}{4 k_{1212}}\left(|\phi|^{2}-v^{2}\right)=0,
\end{aligned}
$$

The equations (24) are identical in form to the Bogomolnyi equations of the abelian Maxwell-Higgs model. The difference lies in the fact that our vortices not only carry magnetic flux, as in the Higgs model, but also electric charge. This is a consequence that in our theory the dynamics of gauge field is dictated by a $C P T$-even Lorentzviolating term instead of a Maxwell term as in Higgs theory.

\section{Self-Dual Chern-Simons vortices without elec- tric charge from $C P T$-odd Lorentz-violating model}

In this section, we analyze the $C P T$-odd Lorentz-violating model [5], coupled to Higgs field

$$
S=\int d^{4} x\left[\frac{1}{4} p_{\alpha} \epsilon^{\alpha \beta \mu \nu} A_{\beta} F_{\mu \nu}+\left|D_{\mu} \phi\right|^{2}+V(|\phi|)\right],
$$

where the coupling coefficient $p_{\alpha}$ is real and has dimensions of mass. The antisymmetric tensor $\epsilon^{\alpha \beta \mu \nu}$ is the totally antisymmetric tensor such that $\epsilon^{0123}=1$.

By varying with respect to $A_{3}$ and $A_{0}$, we obtain the field equations

$$
\begin{aligned}
& p_{\alpha} \frac{1}{2} \epsilon^{\alpha 3 \mu \nu} F_{\mu \nu}+J_{3}=0, \\
& p_{\alpha} \frac{1}{2} \epsilon^{\alpha 0 \mu \nu} F_{\mu \nu}+J_{0}=0,
\end{aligned}
$$

where $J_{0}=-i e\left[\phi^{*}\left(D_{0} \phi\right)-\phi\left(D_{0} \phi\right)^{*}\right]$ and $J_{3}=i e\left[\phi^{*}\left(D_{3} \phi\right)-\phi\left(D_{3} \phi\right)^{*}\right]$.

Here, we are interested in the analysis of the structure of first-order Bogomolnyi self-duality equations for $p_{i}=0(i=1,2,3)$ and $p_{0} \neq 0$. This condition implies a reduction of the action (25) and the equations (26), (27)

$$
\begin{gathered}
S=\int d^{4} x\left[\frac{1}{4} p_{0} \epsilon^{0 \beta \mu \nu} A_{\beta} F_{\mu \nu}+\left|D_{\mu} \phi\right|^{2}+V(|\phi|)\right], \\
p_{0} F_{12}+J_{3}=0 \\
J_{0}=0
\end{gathered}
$$


We can proceed similarly to previous section and reduce one dimension by assuming that the fields do not depend on one of the spatial coordinates, say $x_{3}$, and renaming $A_{3}$ as $N$,

$$
\begin{aligned}
S_{r}= & \int d^{3} x\left(\frac{1}{4} p_{0}\left(2 A_{1} \partial_{2} N-2 A_{2} \partial_{1} N+2 N F_{12}\right)\right. \\
& \left.+\left|D_{0} \phi\right|^{2}-\left|D_{i} \phi\right|^{2}-e^{2} N^{2}|\phi|^{2}+V(|\phi|)\right),
\end{aligned}
$$

Integrating by parts the two first terms of this action, we have,

$$
S_{r}=\int d^{3} x\left(N p_{0} F_{12}+\left|D_{0} \phi\right|^{2}-\left|D_{i} \phi\right|^{2}-e^{2} N^{2}|\phi|^{2}+V(|\phi|)\right),
$$

The equation (29) becomes

$$
N=\frac{p_{0} F_{12}}{2 e^{2}|\phi|^{2}}
$$

Substitution of this equation into the expression of the action (32) yields

$$
S_{r}=\int d^{3} x\left(\frac{p_{0}^{2} F_{12}^{2}}{4 e^{2}|\phi|^{2}}+\left|D_{0} \phi\right|^{2}-\left|D_{i} \phi\right|^{2}+V(|\phi|)\right),
$$

For the static field configuration the equation (30) reads

$$
2 e^{2} A_{0}|\phi|^{2}=0
$$

Thus, the action (34) may be rewritten as

$$
S_{r}=\int d^{2} x\left(\frac{p_{0}^{2} F_{12}^{2}}{4 e^{2}|\phi|^{2}}-\left|\left(D_{1} \pm i D_{2}\right) \phi\right|^{2} \pm e F_{12}|\phi|^{2}+V(|\phi|)\right),
$$

where the identity (10) was used .

To uncover the Bogomolnyi-style self-duality, the action is expressed in the form

$$
\begin{aligned}
S_{r}=\int d^{2} x( & {\left[\frac{p_{0}}{2 e|\phi|} F_{12} \mp \frac{e^{2}}{p_{0}}|\phi|\left(v^{2}-|\phi|^{2}\right)\right]^{2}+\left|i\left(D_{1} \pm i D_{2}\right) \phi\right|^{2} } \\
& \left. \pm e v^{2} F_{12}+V(|\phi|)-\frac{e^{4}}{p_{0}^{2}}|\phi|^{2}\left(v^{2}-|\phi|^{2}\right)^{2}\right),
\end{aligned}
$$

Thus, if the potential is chosen to take the self-dual form

$$
V(|\phi|)=\frac{e^{4}}{p_{0}^{2}}|\phi|^{2}\left(v^{2}-|\phi|^{2}\right)^{2},
$$

the action is bounded below [choosing signs depending on the sign of the flux]

$$
S_{r} \geq e v^{2}|\Phi|
$$


Therefore, when the action reaches its minimum value, the fields will extremise the action and satisfy the first-order field equations

$$
\begin{aligned}
& \left(D_{1} \pm i D_{2}\right) \phi=0 \\
& F_{12}= \pm \frac{2 e^{3}|\phi|^{2}}{p_{0}^{2}}\left(v^{2}-|\phi|^{2}\right),
\end{aligned}
$$

These equations may be compared with the self-duality equations of the Chern-Simons Higgs theory. If we choose $p_{0}$ as the Chern-Simons coupling parameter $\kappa$, the equations (40) coincide with the well know Bogomolnyi equations of the relativistic ChernSimons-Higgs model. Nevertheless, the vortex solutions of the equation (40) are not the identical to those of the relativistic Chern-Simons-Higgs theory. This is because the electric charge density for our solitons is zero, as shown in equation (30).

Finally, it is interesting to note, that in our model the Chern-Simons field does not lead to the equation of the type $E_{i} \propto \epsilon_{i j} J^{j}$, which is an equation of the Chern-Simons-Higgs theory. This is due to that we have considered $p_{i}=0$.

Another difference with the self-dual Chern-Simons-Higgs vortices lies in the fact that in our model the charge density is not locally proportional to the magnetic field, as shown the equations (29) and (30). Thus, in the case of a static field configuration, we should infer the behavior of the field $A_{0}$ from the equation (35). The equation (35) shows that $A_{0}$ may be different from zero if and only if $\phi(x)=0$. Since, our vortex solutions are determined by equations (40), we know, from the Chern-Simons-Higgs solutions, that $\phi(x)$ has an only one root at $x=0$. Therefore, $A_{0}$ may be different from zero, only, at $x=0$. Nevertheless, in order to ensure the regularity of the electric field at $x=0$, we should impose that $A_{0}(0)=0$. Thus, our vortex solution has no electric field.

In summary we have discussed the Bogomolnyi framework for two models with Lorentz breaking symmetry. In the first case we have analyzed the dimensional reduction of a $C P T$-even Lorentz-violating term coupled to Higgs field, showing that the model supports vortex solutions which are identical to those of Maxwell-Higgs model. In the second situation we deal with a reduction of a Carroll-Field-Jackiw term coupled to matter. We have shown, here, that the Bogomolnyi equations of Chern-Simons Higgs theory are obtained as a particular case. It is important to note that in our first model, the vortex solutions has electric charge. This is a difference with the Nielsen-Olesen solutions. Our second model does not have electric charge, being this a difference with relativistic Chern-Simons-Higgs solitons. Finally, we want to note that vortices with electric charge in the CPT-even Lorentz-violating model coupled to matter, were found in Ref.[15]. However the authors use a very strange potential term to generate these solutions, and the self-duality equations differ absolutely from our result.

\section{Acknowledgements}


I would like to thank Rodolfo Casana for useful comments. Also, I would like to thank Dmitri Vassilevich for hospitality during the realization of this work. I am grateful to the referee for his careful reading of the manuscript. This work is supported by CAPES (PNPD/2011).

\section{References}

[1] For review see, V.A. Kostelecky (Ed.), CPT and Lorentz Symmetry III, World Scientific, Singapore, 2005.

V.A. Kostelecky. and S. Samuel, Phys. Rev. D 39, 683 (1989);

V.A. Kostelecky and R. Potting, Nucl. Phys. B 359, 545 (1991).

[2] V.A. Kostelecki and R. Potting, Phys. Rev. D 51, 3923 (1995).

[3] D. Colladay, V.A. Kostelecky, Phys. Rev. D 55, 6760 (1997);

D. Colladay, V.A. Kostelecky, Phys. Rev. D 58, 116002 (1998).

[4] V.A. Kosteleckf, Phys. Rev. D 69, 105009 (2004).

[5] S.M. Carroll, G.B. Field, R. Jackiw, Phys. Rev. D 41 (1990) 1231.

[6] R. Jackiw, V.A. Kostelecky, Phys. Rev. Lett. 82, 3572 (1999);

M. Perez-Victoria, Phys. Rev. Lett. 83, 2518 (1999);

B. Altschul, Phys. Rev. D 70, 101701(R) (2004).

[7] F.R. Klinkhamer, Nucl. Phys. B 578, 277 (2000).

[8] C. Adam, F.R. Klinkhamer, Nucl. Phys. B 607 (2001).

[9] H. Belich, et al., Phys. Rev. D 68, 065030 (2003).

[10] B. Feng, et al., Phys. Rev. Lett. 96, 221302 (2006).

[11] R. Lehnert, R. Potting, Phys. Rev. Lett. 93 (2004) 110402, hep-ph/ 0406128;

R. Lehnert, R. Potting, Phys. Rev. D 70 (2004) 125010, hep-ph/0408285;

C. Kaufhold, F.R. Klinkhamer, Nucl. Phys. B 734 (2006) 1, hep-th/ 0508074.

[12] R. Jackiw, S.-Y. Pi, Phys. Rev. D 68, 104012 (2003). 
[13] M.D. Seifert, Phys. Rev. D 82, 125015 (2010).

M.D. Seifert, Phys. Rev. Lett. 105, 201601 (2010)

[14] C. Miller, R. Casana, M.M. Ferreira, Jr., E. da Hora Phys.Rev. D 86065011 (2012);

[15] C. Casana, M.M. Ferreira, Jr., E. da Hora, C. Miller Phys.Lett. B 718 620-624 (2012);

[16] R. Casana, L. Sourrouille, Physics Letters B, 726:488492,(2013), [arXiv:hepth/1307.1734].

[17] H.B. Nielsen, P. Olesen, Nucl. Phys. B 6145 (1973).

[18] E. Bogomolyi, Sov. J. Nucl. Phys 24, 449 (1976);

H. de Vega and F .A. Schaposnik, Phys. Rev. D 14, 1100 (1976).

[19] E. Witten and D. I. Olive, Phys. Lett. B 78 (1978) 97.

[20] S. K. Paul and A. Khare, Phys. Lett. B 174, 420 (1986) [Erratum-ibid. 177B, 453 (1986)];

H. J. de Vega and F. A. Schaposnik, Phys. Rev. D 34, 3206 (1986)

[21] R. Jackiw and E. Weinberg, Phys. Rev. Lett. 642334 (1990).

J. Hong, Y. Kim and P.-Y. Pac, Phys. Rev. Lett. 642230 (1990).

[22] R. Jackiw and S. Y. Pi, Phys. Rev. Lett. 64, 2969 (1990); R. Jackiw and S. Y. Pi, Phys. Rev. D 42, 3500 (1990). [Erratum-ibid. D 48, 3929 (1993)].

[23] R. Jackiw, Ki-Myeong Lee and E.J. Weinberg, Phys.Rev. D 42, 3488 (1990). 